\title{
Caracterización de la población con epilepsia atendida en un hospital infantil en Manizales 2013-2014
}

Characterization of patients with epilepsy treated in a children's hospital in Manizales 2013-2014

José Jaime Castaño-Castrillón ${ }^{1 \otimes C \text { CvLAC, }}$, Yesenia Alejandra Campos-Quimbayo ${ }^{2 \otimes}$, Carlos Alberto DuqueJiménez ${ }^{2}$, Erika Grisales-Aristizábal², Tatiana María Mera-Romo², Gustavo Adolfo Rodríguez-Alzate ${ }^{2}$, Oscar Alberto Villegas-Arenas ${ }^{3 \otimes \text { CvLAC }}$

Fecha correspondencia: Recibido: diciembre 28 de 2015. Revisado: junio 14 de 2016. Aceptado: julio 21 de 2016.

\section{Forma de citar:}

Castaño-Castrillón JJ, CamposQuimbayo YA, Duque-Jiménez CA, Grisales-Aristizábal E, Mera-Romo TM, RodríguezAlzate GA, Villegas-Arenas OA. Caracterización de la población con epilepsia atendida en un hospital infantil en Manizales 2013-2014. Rev CES Med 2016; 30(2): 139-147.

\section{$\underline{\text { Open access }}$}

(c) Copyright

Licencia creative commons

Ética de publicaciones

Revisión por pares

Gestión por Open Journal System

ISSN 0120-8705

e-ISSN 2215-9177

Comparte

\section{Resumen}

Objetivo: caracterizar la población con diagnóstico de epilepsia en el Hospital Infantil Universitario Rafael Henao Toro, en Manizales (Colombia), desde el punto de vista del comportamiento de la enfermedad, factores de riesgo, tratamiento y particularidades demográficas. Métodos: estudio de corte transversal realizado mediante la revisión de historias clínicas. Resultados: de 172 pacientes, 51,2 \% fueron del género masculino; las crisis focales fueron las más frecuentes con 59,1\%; la comorbilidad más usual fue el retraso del desarrollo psicomotor (30,9\%), aunque si se tiene en cuenta la asociación con otras enfermedades la cifra asciende al 57,9 \%; la monoterapia más utilizada fue el ácido valpróico (39,8 \%). La epilepsia infantil se asoció con estados hipertensivos maternos en un 8,7 \%; el 21,7 \% presentó encefalopatía hipóxica-isquémica; 24,1 \% tenían antecedentes familiares de epilepsia; en 15,1\% de los casos hubo bajo peso y $16,7 \%$ de talla baja al nacer. Conclusiones: los hallazgos de la presente investigación, en general, coinciden con lo reportado en otros estudios efectuados en poblaciones análogas.

Palabras clave: Epilepsia, Encefalopatía hipóxica-isquémica, Anticonvulsivantes, Comorbilidad, Factores de riesgo.

\footnotetext{
Abstract

Objective: To characterize the diagnosed with epilepsy population at the "Hospital Infantil Universitario Rafael Henao Toro", in the city of Manizales (Colombia) from the behavior of the disease, risk factors, treatment and demographic characteristics. Methods: Cross-sectional study of the population referred to and performed by reviewing medical records. Results: of 172 patients, $51,2 \%$ were male gender; focal seizures were the most frequent with $59.1 \%$; the most common comorbidity was delayed psychomotor development (30.9\%), although if is consider the association with other diseases the figure is $57.9 \%$; the most commonly monotherapy used was Valproic Acid (39.8\%). Childhood epilepsy was associated with maternal hypertensive disorders in $8,7 \% ; 21.7 \%$ had hypoxic-ischemic encephalopathy; $24.1 \%$ had a family history of epilepsy; in $15.1 \%$ of cases was under-
} 


\author{
Sobre los autores: \\ 1 Profesor Titular, Director \\ Grupo de Investigación \\ Médica, Facultad de Ciencias \\ de la Salud, Universidad de \\ Manizales. \\ 2 Estudiante de Medicina, \\ Universidad de Manizales. \\ 3 MD, Mg, Profesor asociado, \\ Programa de Medicina, \\ Universidad de Manizales .
}

Se estima que en el mundo hay 50 millones de personas con epilepsia, $80 \%$ de ellas viven en países pobres y de estos, unos ocho millones están en Latinoamérica y el Caribe. weight and $16.7 \%$ of short stature at birth. Conclusion: The findings of this research, in general, agree with those reported in other studies in similar populations.

Key words: Epilepsy, Brain hypoxia-ischemia, Anticonvulsants, Comorbidity, Risk factors

\section{Introducción}

La epilepsia es uno de los trastornos más frecuentes en neurología y envuelve varios tipos de compromiso y síndromes epilépticos (1). Es definida como un desorden cerebral caracterizado por una predisposición duradera a generar convulsiones epilépticas asociadas a consecuencias neurobiológicas, cognitivas, psicológicas y sociales (1- $\underline{3}$. Se considera que una persona tiene epilepsia cuando presenta estos episodios no originados por alteraciones médicas identificadas como: convulsiones febriles, abstinencia al alcohol u otras drogas, alteraciones metabólicas, entre otras $(\underline{1}, \underline{4})$.

Se estima que en el mundo hay 50 millones de personas con epilepsia, 80 \% de ellas viven en países pobres y de estos, unos ocho millones están en Latinoamérica y el Caribe (5). Cerca del $3 \%$ de la población tendrá diagnóstico de epilepsia en algún momento de su vida (6, 7). Son reportadas prevalencias de 5,7 a 6,8 y de 4,0 por cada mil habitantes para Estados Unidos y el Reino Unido, respectivamente (1, 6,, 7). Para Colombia fue de 21,4 por 1000 habitantes en 1983, mientras que en otro estudio en 1996 se encontró en 22,7 por cada 1000 habitantes en el oriente colombiano (8)). Para el departamento de Caldas, Díaz et al. () hallan prevalencia de 24 por 1000 habitantes; lo propio hace Alcázar et al. (10) para Manizales en 2008 (17\%00).

Ríos et al. (11), Glass et al. (12), Whitehead et al. (13) y Sen et al. (14) encuentran que la encefalopatía hipóxico-isquémica o posasfíctica y los estados hipertensivos en el embarazo tienen relación con la presencia de epilepsia en los niños (15). Para Knupp et al. (16) y Ortiz (17), los antecedentes familiares para epilepsia son también factores de riesgo asociados (18). Las neuroinfecciones (19), los traumatismos craneoencefálicos y anomalías del sistema nervioso central $(\underline{9}, 20)$, se relacionan con la presencia de convulsiones provocadas (no epilépticas). Jeldres et al. (21) en su estudio con 239 niños epilépticos, encuentran que un $80 \%$ conviven con una o más enfermedades asociadas a la epilepsia.

Esta investigación tuvo como objetivo conocer aspectos relacionados con el comportamiento de la epilepsia en la población pediátrica consultante en el Hospital Infantil de Manizales (Colombia) y su relación con los factores de riesgo perinatales, antecedentes familiares, tipo de crisis, tratamientos instaurados y comorbilidades.

\section{Metodología}

Estudio descriptivo empleando 172 historias clínicas de pacientes pediátricos con diagnóstico de epilepsia y consultantes al Hospital Infantil Universitario Rafael Henao Toro de la Cruz Roja en Manizales (Colombia), entidad de salud de carácter privado, que brinda servicios de tercer nivel de complejidad a pacientes de la ciudad y del departamento de Caldas, durante el periodo de 2013 y 2014.

Los sesgos internos se minimizaron al tener en cuenta la información de la totalidad de casos registrados en la atención ambulatoria, independiente de si eventualmente consultaron en urgencias o fueron internados. Dos instituciones de salud no participaron de la investigación. 
La edad promedio de diagnóstico fue a los $5,4 \pm 3,8$ años y el tipo de crisis más frecuente fue la crisis focal con un $59,1 \%$, mientras que las crisis generalizadas representan un $29,8 \%$ y las crisis indeterminadas $11,1 \%$.
El presente estudio utilizó la clasificación internacional de los síndromes epilépticos de la International League Against Epilepsy (ILAE) de 1989, que incluye convulsiones parciales, convulsiones generalizadas típicas y atípicas, convulsiones generalizadas tónicas y clónicas, convulsiones focales, espasmo epiléptico, estatus no convulsivos, estatus focales, estatus epilepticus (1- $\underline{3})$.

Criterios de inclusión: historias clínicas completas de todos los niños con diagnóstico de epilepsia realizadas por el neurólogo pediatra y tratados en el Hospital infantil de Manizales. Los criterios de exclusión fueron: casos de niños con convulsiones que no cumplieron como síndromes epilépticos, tales como epilepsia parcial rolándica benigna y la epilepsia ausencia, síndrome de Lennox-Gastaut, síndrome de West entre otros $(\underline{2}, \underline{3})$. Todas las variables se obtuvieron de las historia clínicas.

Los análisis estadísticos de las variables nominales se describieron mediante tablas de frecuencia y límites de confianza del $95 \%$, y las variables numéricas mediante promedios, desviaciones estándar, con límites de confianza del $95 \%$. La relación entre variables medidas en escala nominal se determinó empleando el procedimiento estadístico de $\mathrm{X}^{2}$ y entre variables medidas en escala nominal y de razón mediante prueba t de Student o análisis de varianza, según el caso. Todos los análisis de relación se efectuaron con una significancia de 0,05. La base de datos se elaboró en el programa Excel 2010 (Microsoft Corporation) y se analizó empleando el programa IBM SPSS $21{ }^{\circledR}$ (IBM Corp.).

El proyecto fue aprobado por el Comité de Ética e Investigación de la IPS y por el tipo de estudio y fuente de datos, corresponde a una investigación sin riesgo.

La clasificación de los grupos se basó en el siguiente rango etario: neonato, de 0 a 28 días; lactante menor, de 1 a 11 meses; lactante mayor, de 12 a 23 meses; preescolar, de 2 a 5 años; escolar, de 6 a 10 años; adolescencia temprana, de 11 a 13 años; media, de 14 a 16 años y tardía, de 17 a 19 años (22).

\section{Resultados}

Se observó un predominio del género masculino (51,2 \%) y el lugar de residencia más frecuente fue el urbano (84,9\%); el 55,2 \% procedían de Manizales. La asistencia a los controles prenatales fue de $96,6 \%$ y el lugar de parto fue institucional $(97,4 \%)$. El parto vaginal sucedió en 78,4 \% de los casos. La proporción de preeclampsia fue de 8,7 \%; el $21,7 \%$ presentó encefalopatía hipóxica-isquémica y en el $24,1 \%$ se identificaron antecedentes familiares de epilepsia.

En promedio, la edad gestacional fue de 38,2 $\pm 2,4$ semanas, el 11,3\% fueron pretérmino; el peso promedio al nacer fue de $3021 \pm 642 \mathrm{gr}$, el 15,1\% presentaron bajo peso al nacer; la talla promedio al nacer fue de $48,39 \pm 3,4 \mathrm{~cm}$ y el $16,7 \%$ tuvieron talla baja.

En el cuadro 1 se hace referencia a las características de la epilepsia. Se observa que la edad promedio de diagnóstico fue a los 5,4 \pm 3,8 años y el tipo de crisis más frecuente fue la crisis focal con un 59,1\%, mientras que las crisis generalizadas representan un $29,8 \%$ y las crisis indeterminadas $11,1 \%$.

El 52,3\% presentaron comorbilidades y de estos el 56,6 \% presentaron una comorbilidad. La comorbilidad más frecuente fue el retraso en el desarrollo psicomotor con un $30,9 \%$ si se considera individualmente; pero cuando se acompaña de otras comorbilidades, la cifra llega al 57,9\%. 
La monoterapia más empleada fue el ácido valpróico que se usó en el 39,8\%; la cifra asciende al $48 \%$ si se tienen en cuenta las terapias combinadas.
Cuadro 1. Características de los episodios epilépticos

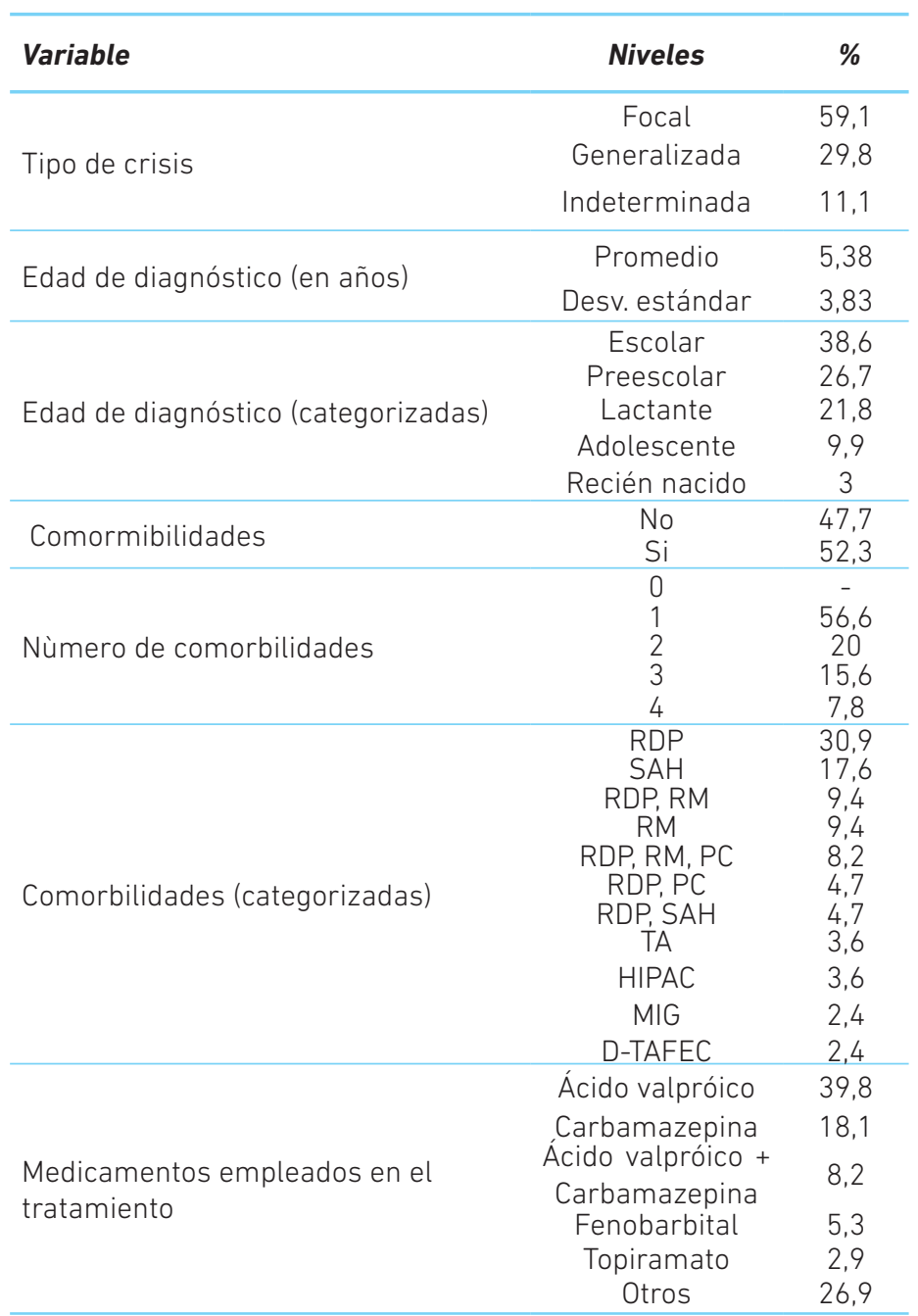

RDP: retraso desarrollo psicomotor, SAH: síndrome de atención e hiperactividad, RM: retardo mental, PC: parálisis cerebral, TA: trastorno autista, HIPAC: hiperactividad, MIG: migraña, DTAFEC: depresión-trastorno afectivo.

La monoterapia más empleada fue el ácido valpróico que se usó en el 39,8%; la cifra asciende al $48 \%$ si se tienen en cuenta las terapias combinadas.

El ácido valpróico fue usado principalmente en las crisis generalizadas (84,2 \%), carbamazepina en las focales (45\%), topiramato $(36,8 \%)$ y fenobarbital en las indeterminadas (21,1\%) (cuadro 2).

Se encontró relación entre tipo de crisis y otras variables cuantificadas, entre otras las comorbilidades presentadas. Las relaciones significativas encontradas se presentan en el cuadro 3.

\section{Discusión}

Referente a la edad, Dura (23) en 2007 reporta una media de 5,9 años para hacer el diagnóstico de epilepsia y la distribución por grupos de edad más frecuente fue la preescolar, hallazgo similar el encontrado en la presente investigación. En el estudio realizado por Whitehead et al. (13) en 648 pacientes con epilepsia, la media de edad de diagnóstico fue de 8,5 años. En la investigación de Wagner et al. (24) encuentran que la mayor incidencia de epilepsia ocurre en niños menores de cinco años, dato 
Cuadro 2. Medicamentos empleados según el tipo de crisis

\begin{tabular}{lccccc}
\hline Tipo de crisis (\%) & $\begin{array}{c}\text { Ácido } \\
\text { valpróico } \\
\boldsymbol{p = 0 , 0 0 0}\end{array}$ & $\begin{array}{c}\text { Topiramato } \\
\boldsymbol{p = 0 , 0 0 0}\end{array}$ & $\begin{array}{c}\text { Carbamazepina } \\
\boldsymbol{p = 0 , 0 0 0}\end{array}$ & $\begin{array}{c}\text { Vigabatrina } \\
\boldsymbol{p = 0 , 0 0 0}\end{array}$ & $\begin{array}{c}\text { Fenobarbital } \\
\boldsymbol{p = 0 , 0 2 3}\end{array}$ \\
\hline Indeterminada & 15,8 & 36,8 & 15,8 & 36,8 & 21,1 \\
Focal & 58 & 5 & 45 & 1 & 5 \\
Generalizada & 84,2 & 2 & 23,7 & 0 & 3,9 \\
\hline
\end{tabular}

Cuadro 3. Relación entre tipo de crisis y otras variable

\begin{tabular}{|c|c|c|c|c|c|}
\hline \multirow{2}{*}{ Variable } & \multirow[b]{2}{*}{ Niveles } & \multicolumn{4}{|c|}{ Tipos de crisis (\%) } \\
\hline & & $I$ & $F$ & G & Valor $p$ \\
\hline \multirow{2}{*}{ Vía del parto } & $\begin{array}{c}\text { Cesárea } \\
n=32\end{array}$ & 15,2 & 72,7 & 12,1 & \multirow{2}{*}{0,013} \\
\hline & $\begin{array}{c}\text { Vaginal } \\
n=119\end{array}$ & 7,6 & 53,8 & 38,7 & \\
\hline \multirow[b]{2}{*}{ Encefalopatía hipóxica-isquémica } & $\begin{array}{c}\text { No } \\
n=107\end{array}$ & 5,6 & 5,7 & 37,4 & \multirow[b]{2}{*}{0,000} \\
\hline & $\begin{array}{c}\mathrm{Si} \\
\mathrm{n}=30\end{array}$ & 33,3 & 53,3 & 13,3 & \\
\hline \multirow{2}{*}{ Retraso del desarrollo psicomotor } & $\begin{array}{c}\text { No } \\
n=103\end{array}$ & 6,9 & 56,9 & 36,3 & \multirow{2}{*}{0,009} \\
\hline & $\begin{array}{c}\mathrm{Si} \\
\mathrm{n}=52\end{array}$ & 21,2 & 59,6 & 19,2 & \\
\hline \multirow{2}{*}{ Trastorno autista } & $\begin{array}{c}\text { No } \\
n=151\end{array}$ & 11,3 & 57,6 & 31,1 & \multirow{2}{*}{0,014} \\
\hline & $\begin{array}{c}\mathrm{Si} \\
\mathrm{n}=3\end{array}$ & 66,7 & 33,3 & 0 & \\
\hline \multirow{2}{*}{ Parálisis cerebral } & $\begin{array}{c}\text { No } \\
n=138\end{array}$ & 10,1 & 55,8 & 34,1 & \multirow{2}{*}{0,011} \\
\hline & $\underset{n=16}{S i}$ & 25 & 75 & 0 & \\
\hline
\end{tabular}

I: Indeterminada F: focales G: generalizadas

que es consistente con nuestra caracterización, cuando se tienen en cuenta conjuntamente los lactantes y los preescolares (48,5\%).

Alcázar et al. (10) en 2008 en Manizales, en un grupo de niños, encuentran que el género masculino $(61,3 \%)$ presenta más crisis epilépticas; esta distribución por género también se encuentra en el estudio realizado por Sun et al. (25) con 16455 niños, así como que el 53,1 \% eran varones. Hawley et al. (26) reportan mayor prevalencia en niños que en niñas, aunque sin diferencias estadísticas.

Sun et al. (25) y Alcázar et al. (10) encuentran que la procedencia urbana es mayoritaria en ambos casos con un 60,8 \% y para la presente investigación es mucho más elevada, en el $84,9 \%$.

Sun et al. (25), describen que el tipo de crisis más común son las generalizadas (65,3 \%), así como Martínez et al. (27), quienes hallan más frecuentes las crisis generalizadas seguidas por las focales, que en la presente investigación ocurrieron en el 59,1\% de los niños coincidente con Carrizosa (ㅇ) quien en 2007 encontró también que el tipo de crisis más frecuente es la focal (64\%). 
Los niños con encefalopatía hipóxica-isquémica tienen cinco veces más probabilidad de desarrollar epilepsia que los no expuestos a dicha condición.
En el estudio sobre crisis convulsivas en 138 neonatos, Ríos et al. (11) documentan la encefalopatía hipóxico-isquémica como el mayor agente etiológico responsable en $34,8 \%$ de los casos y Glass et al. (12) reportan que la incidencia de esta misma entidad es de aproximadamente 0,1 a $0,25 \%$ de los nacidos vivos a término, como consecuencia de la asfixia perinatal.

En la presente investigación se documenta este antecedente en 21,7 \% de los sujetos, lo que sugiere que existe una relación entre los eventos isquémicos perinatales y el riesgo de generar convulsiones o epilepsia; tal como lo afirma la literatura médica (28). Los niños con encefalopatía hipóxica-isquémica tienen cinco veces más probabilidad de desarrollar epilepsia que los no expuestos a dicha condición. La tasa reportada de epilepsia después de encefalopatía hipóxico-isquémica oscila entre 9 y $33 \%(\underline{15}, \underline{25}, \underline{26})$.

En el trabajo de Sun (25) et al., el retraso mental es la comorbilidad que más se presenta (34,3\%). Un estudio sobre comorbilidades en 239 niños con epilepsia en Chile encuentra que un $80 \%$ tienen una o más comorbilidades asociadas a la epilepsia (21). Las más frecuentemente detectadas son el retraso del desarrollo psicomotor/ retraso mental, trastorno por déficit atencional, parálisis cerebral y retraso del lenguaje. En el presente trabajo se encontró que un 52,3 \% de los pacientes contaban con alguna comorbilidad.

Sen et al. (14) encuentran que el 2,9\% de los pacientes epilépticos estuvieron expuestos a preeclampsia y el 0,04\% a eclampsia en su vida prenatal. Y para el presente estudio lo fue en el 8,7\%, es decir, podría ser un factor de riesgo de importancia. El bajo peso al nacer hallado es mayor al encontrado en otra investigación en una población general similar $(4,4 \%)(\underline{28})$, lo que permite relacionar esta condición con la epilepsia infantil. Carrascosa et al. (ㅇ) hacen un análisis prospectivo de peso, talla y perímetro cefálico al nacimiento, en 1786 recién nacidos vivos en el Hospital Materno-Infantil Vall d'Hebron de Barcelona en gestaciones únicas y a término y en 1470 recién nacidos vivos de gestaciones únicas pretérmino, encontrando mayor riesgo de adquirir condiciones patológicas en estos últimos (29).

Según Knupp et al. (16) los datos epidemiológicos sugieren un riesgo inicial para la epilepsia de $1 \%$ en la población general. Otros estudios han encontrado un riesgo de 2 a $4,6 \%$ para los individuos que tiene un familiar epiléptico en primer grado $(\underline{8}, \underline{18})$.

Aunque esto representa una duplicación del riesgo, más del $75 \%$ de los individuos con antecedentes familiares positivos tienen un solo pariente afectado y algunas familias siguen patrones mendelianos de herencia. Este patrón implica una forma compleja de herencia, cuyo riesgo es impartido por los efectos aditivos de múltiples genes y factores ambientales $(\underline{13}, \underline{18})$.

El presente estudio arrojó que casi una cuarta parte de los pacientes estudiados $(24,1 \%)$ presentaba un familiar hasta de segundo grado con antecedente de epilepsia. En el trabajo de Wagner et al. (24), la historia familiar de epilepsia y tener un hermano con epilepsia es factor de riesgo significativo para epilepsia. Adicionalmente, Chentouf et al. (18) en su estudio encuentran datos muy significativos con respecto a familiares de primer grado con epilepsia como gran factor de riesgo.

Acevedo et al. (므) describen que los medicamentos de primera elección para el tratamiento de las crisis focales, son la fenitoína y la carbamazepina; para las crisis 
Esta investigación coincide con la literatura mundial en cuanto a que el factor de riesgo principal es la encefalopatía hipóxicaisquémica o postasfíctica, aunque con trascendente exposición a la preeclampsia, historia familiar de epilepsia y bajo peso al nacer. generalizadas es el ácido valpróico, siendo el fenobarbital una muy buena opción, en terapia combinada con carbamazepina para crisis focales y como alternativa se usa el ácido valpróico como monoterapia para crisis generalizadas (ㅁ).

En el presente estudio se encontró que en general el medicamento más empleado fue el ácido valpróico como monoterapia (39,8 \%) y como terapia combinada (48\%), seguido de la carbamazepina, y dentro de los terapias combinadas la más utilizada fue ácido valpróico más carbamazepina. Para las crisis focales, el ácido valpróico se usó más que la carbamazepina y si de crisis generalizada se trata, fue el preferido con una diferencia más significativa. Chentouf et al. (18) reportan que el agente antiepiléptico más usado es el ácido valpróico (31,7 \%), similar en el presente estudio.

Esta investigación coincide con la literatura mundial en cuanto a que el factor de riesgo principal es la encefalopatía hipóxica-isquémica o postasfíctica, aunque con trascendente exposición a la preeclampsia, historia familiar de epilepsia y bajo peso al nacer, es decir, estos son los factores de riesgo más significativos asociados a esta enfermedad, lo que puede motivar al grupo de trabajo u otros, seguir investigando desde estos puntos de vista sobre el tema.

Como limitaciones del estudio se identifican que solo se contó con los pacientes pediátricos atendidos en el Hospital Infantil Rafael Henao Toro y que no fue posible recolectar todas las variables que se habían planteado para la investigación, por omisión de valiosos datos en la historia clínica.

Sería ideal disponer en Manizales de una clínica especializada en epilepsia, que optimizaría el recurso, con mejores logros en la prevención, la detección y el tratamiento; también para mejorar la capacitación e investigación.

\section{Conflictos de interés}

Ninguno.

\section{Fuentes de financiación}

Universidad de Manizales.

\section{Bibliografía}

1. Engel J Jr. Epilepsy; Overview. $2^{\circ}$ ed. Oxford: Academic Press; 2014.

2. Fisher RS, Acevedo C, Arzimanoglou A, Bogacz A, Cross JH, Elger CE, et al.. ILAE official report: a practical clinical definition of epilepsy. Epilepsia 2014; 55(4):47582. https://www.ncbi.nlm.nih.gov/pubmed/24730690

3. Fisher RS, Acevedo C, Arzimanoglou A, Bogacz A, Cross JH, Elger C, et al. An operational clinical definition of epilepsy. Palo Alto: International League Against Epilepsy; 2013. http://www.ilae.org/visitors/centre/documents/DefinitionCommentsHBester.pdf

4. Martínez M, Masakazu S, Munari C, Porter R, Roger J, Wolf P, et al. Commission on classification and terminology of the International League Against Epilepsy. proposal for revised classification of epilepsies and epileptic syndromes. Epilepsia 1989; 30(4):389-99. https://www.ncbi.nlm.nih.gov/pubmed/2502382 
5. Acevedo C, Miranda C, Campos M, Caraballo R, Carpio A, Cuadra L, et al. Informe sobre la epilepsia en Latinoamérica OPS/OMS. Ciudad de Panamá: AG Publicidad; 2008. http://www.ilae.org/visitors/policy/documents/PAHO-report2013-Spanish. pdf

6. Sander JW. Epilepsy; Epidemiology. $2^{\circ}$ ed. Oxford: Academic Press; 2014.

7. Cansu A, Serdaroğlu, A, Yüksel D. Prevalence of some risk factors in children with epilepsy compared to their controls. Seizure 2007; 16(4):338-44. https:// www.ncbi.nlm.nih.gov/pubmed/17391991

8. Carrizosa J. Prevalencia, incidencia y brecha terapéutica en la epilepsia. latreia 2007; 20(3): 282-296. http://www.redalyc.org/pdf/1805/180513858006.pdf

9. Díaz R, Ruano M, Chacón J, Vera A. Perfil Neuroepidemiológico en la zona centro del Departamento de Caldas (Colombia), años 2004-2005. Rev Neurol 2006; 43 (11): 6-46. http://www.scielo.org.co/scielo.php?script=sci arttext\&pid $=$ S0120-24482009000200003

10. Alcázar J, Castaño J, Fragoso L, García S, Giraldo S, Jiménez S, et al. Factores de riesgo presente en una muestra de población epiléptica de manizales, Caldas, Colombia. Arch Med (Manizales) 2008; 8(1):32-39. http://revistasum.umanizales. edu.co/ojs/index.php/archivosmedicina/article/download/1322/1427

11. Ríos B, Rey L, Ibarra J, Olivas E. Prevalencia de crisis convulsivas neonatales en el Instituto Nacional de Perinatología. Rev Mex Neuroci 2007; 8(4):360-366. http://revmexneuroci.com/wp-content/uploads/2014/06/Nm074-05.pdf

12. Glass H, Hong K, Rogers E, Jeremy R, Bonifacio S, Sullivan J, et al. Risk factors for epilepsy in children with neonatal encephalopathy. Pediatr Res 2011; 70: 535540. https://www.ncbi.nlm.nih.gov/pmc/articles/PMC3189270/

13. Whitehead E, Dodds L, Joseph K, Gordon K, Wood E, Allen A, et al.. Relation of pregnancy and neonatal factors to subsequent development of childhood epilepsy: a populationbased cohort study. Pediatrics 2006; 117: 1297-1307.

14. Sen C, Sun Y, Vestergaard M, Christense J, Ness R, Haggerty C, et al. Preeclampsia and risk for epilepsy in offspring. Pediatrics 2008; 122: 1072-1078. http://pediatrics.aappublications.org/content/122/5/1072?download=true

15. García A, Pérez A. Estado fetal no tranquilizador, asfixia perinatal y encefalopatía neonatal. Ann Pediatr 2005; 63:1-4. http://www.analesdepediatria.org/es/estadofetal-no-tranquilizador-asfixia/articulo/13076760/

16. Knupp K, Koh S, Park K. Pediatric epilepsy: five new things. Neurol Clin Pract 2011; 76(2):s20-s25. http://www.neurology.org/content/76/7 Supplement_2/S20. short

17. Ortiz M. La epilepsia en pediatría, primera de dos partes. Rev Mex Puer Pediatr 2003; 11(61):23-29. 
18. Chentouf A, Talhi R, Dahdouh A, Benbihi L, Benilha S, Oubaiche M, et al. Consanguinity and epilepsy in Oran, Algeria: A case-control study. Epilepsy Res 2015, 111:10-7. https://www.ncbi.nlm.nih.gov/pubmed/25769368

19. Rodríguez L, Quispe Y, Sifuentes J. Factores de riesgo de la epilepsia secundaria en niños. Rev Neuro Psiquiatr 2002; 65:136-141. http://www.upch.edu.pe/vrinve/ dugic/revistas/index.php/RNP/article/view/1516

20. Hesdorffer DC, Hauser WA. Epilepsy; risk factors. $2^{\circ}$ ed. Oxford: Academic Press; 2014.

21. Jeldres E, Devilat M, Peralta S, Gómez V. Comorbilidad en niños con epilepsia. Rev Chil Epilepsia 2010; 2:19-28. http://www.revistachilenadeepilepsia.cl/wp-content/uploads/2014/08/201401 completa.pdf

22. Velásquez OJ. Pediadatos tablas, fórmulas y valores normales en pediatría. $3^{a}$ ed. Bogotá DC: Editorial Hipertexto Ltda; 2011. http://www.libreriadelau.com/pediadatos-tablas-44-formulas-y-valores-normales-en-pediatria-medicina.html

23. Dura T, Petri M, Gallinas F. Estudio descriptivo de la epilepsia infantil. Rev Neurol 2007; 44: 720-724. http://www.neurologia.com/pdf/web/4412/x120720.pdf

24. Wagner RG, Ngugi AK, Twine R, Bottomley C, Kamuyu G, Gómez-Olivé FX. Prevalence and risk factors for active convulsive epilepsy in rural northeast South Africa. Epilepsy Res 2014; 108(4):782-91. https://www.ncbi.nlm.nih.gov/ pubmed/24582322

25. Sun Y. Vestergaard M, Bocker C, Christensen J, Olsen J. Apgar scores and longterm risk of epilepsy. Epidey 2006; 17:296-301. https://www.ncbi.nlm.nih.gov/ pubmed/16570027

26. Hawley SR, Ablah E, Hesdorffer D, Pellock JM, Lindeman DP, Paschal AM, et al. Prevalence of pediatric epilepsy in low-income rural Midwestern counties, Epilepsy Behav 2015; 53:190-6. https://www.ncbi.nlm.nih.gov/pubmed/26588587

27. Martínez X, Duarte Y, Portales G, Mirabal G. Estudio epidemiológico de la epilepsia infantil en el municipio de Bahía Honda. Rev Ciencias Médicas 2011; 15(1):76-88. http://scielo.sld.cu/scielo.php?script=sci abstract\&pid=S1561-31942011000100007

28. Castaño JJ, Giraldo JF, Murillo CA, Jordán Y, Orozco JA, Robledo P, et al. Relación entre peso al nacer y variables biológicas y socioeconómicas de la madre en partos atendidos en un primer nivel de complejidad en la ciudad de Manizales, Colombia, 1999 al 2005. Rev Colomb Obstet Ginecol 2008; 59(1):20-25. http:// www.scielo.org.co/pdf/rcog/v59n1/v59n1a03.pdf

29. Carrascosa A, Yeste D, Copil A, Almar J, Salcedo S, Gussinyé M. Patrones antropométricos de los recién nacidos pretérmino y a término (24-42 semanas de edad gestacional) en el Hospital Materno (Barcelona) (1997-2002). An Pediatr (Barc) 2004; 60(5):406-16. http://www.analesdepediatria.org/es/pdf/S1695403304782995/S300/ 